\title{
In-gap states of the quantum dot coupled between a normal and superconducting lead
}

\author{
J. Barański and T. Domański \\ Institute of Physics, M. Curie-Sktodowska University, 20-031 Lublin, Poland
}

(Dated: June 8, 2021)

\begin{abstract}
We study the in-gap states of the quantum dot hybridized with one conducting and another superconducting electrode. Proximity effect suppresses the electronic states in the entire subgap regime $|\omega|<\Delta$, where $\Delta$ denotes the energy gap of a superconductor. The Andreev scattering mechanism can induce, however, some in-gap states of a line-broadening (inverse life-time) controlled by a hybridization of the quantum dot with a normal electrode. We show that the number of such Andreev bound states is substantially dependent on a competition between the Coulomb repulsion and the induced on-dot pairing. We discuss signatures of these in-gap states in the tunneling conductance, especially in a low-bias regime.

PACS numbers: 73.63.Kv;73.23.Hk;74.45.+c;74.50.+r
\end{abstract}

\section{MOTIVATION}

The quantum impurities embedded in superconducting host materials have been the topic of intensive studies for about 50 years (see the review paper [1]). Early interests predominantly explored in what way the impurities affect the superconducting state of the bulk materials. It has been established (by the Anderson theorem [2]) that the paramagnetic impurities are rather inefficient on the isotropic superconductors or eventually weakly suppress the anisotropic superconducting phases [3]. The magnetic (spinful) impurities, on contrary, proved to have much stronger influence on superconductivity. They induce the in-gap states [4] and with increasing concentration of the magnetic impurities the energy gap of superconducting material is gradually filled-in simultaneously suppressing its critical temperature. On a microscopic level this detrimental effect comes from a pair-breaking character of the spin scattering.

Mutual relationship between the quantum impurities and superconducting materials attracts presently again substantial interests due to intensive studies of the nanoscopic devices, where various artificial quantum impurities (dots) are connected to the external superconducting electrodes. In this context, the main problem refers to the question how do the superconducting reservoirs affect the quantum dots rather than the other way around. Due to the proximity effect the Cooper pairs can penetrate the quantum dot, converting it into a sort of the 'superconducting grain'. On the other hand, the strong Coulomb repulsion between opposite spin electrons disfavors any double (or even) occupancy of the quantum dot. At low temperatures also the Kondo physics additionally comes into the play. Both these phenomena, i.e. the Coulomb blockade and appearance of the Kondo singlet state, strongly compete with the induced on-dot pairing. In nanoscopic tunneling junctions this competition can be explored in a controllable manner, by: a) varying the $\mathrm{QD}$ hybridization with the superconducting lead, b) affecting the energy gap $\Delta=\Delta(B)$ by applying the magnetic field $B$ [5], c) lifting the discrete QD energy levels via the gate voltage, and d) lowering temperatures to activate the Kondo physics. Numerous theoretical and experimental studies of the quantum dots connected to the superconducting leads have been summarized e.g. in Refs [6, 7].

Interplay between the on-dot pairing and the correlation effects can be conveniently investigated in the setup, where the quantum dots are placed between one superconducting (S) and another normal $(\mathrm{N})$ electrode. In the subgap regime $(e V<\Delta)$ the tunneling conductance practically entirely originates from the anomalous Andreev channel, such spectroscopy can thus directly probe any in-gap states. In experimental realizations of the NQD-S junctions the role of quantum dots has been played by the self-assembled InAs nanoscopic islands [8], carbon nanotubes 9], quantum wires [10] etc. For instance, using InAs quantum dots coupled between the metallic (golden) and superconducting (aluminum) electrodes provided a clear evidence for the Kondo effect coexisting with the induced on-dot pairing manifested by the zerobias enhancement of the zero-bias Andreev conductance [8]. Tunneling conductance has been recently measured also in the system comprising the indium antimonide nanowires connected to a normal (gold) and superconducting (niobium titanium nitride) electrode, indicating the Majorana type in-gap states [10].

Other measurements have been done using the threeterminal configurations with the metallic and superconducting electrodes interconnected via the double quantum dots to achieve a controllable Cooper pair splitting. These dots served as 'quantum forks', where the Coulomb repulsion enforced electrons (released from the Cooper pairs) to move into different normal leads, yet preserving their entanglement. Such transport channel contributed about 10 percent (for the case of InAs quantum dots 18]) and nearly 50 percent (using the carbon nanotubes [19]) to the total differential conductance. For the latter case the efficiency has been next considerably improved [20]. In experimental measurements there have also probed the spin-polarized Andreev current using the ferromagnetic electrode coupled via the quantum dots to the superconducting lead 21]. In all these and many other related experiments [22, 23] the subgap electron transport is solely 
provided by the in-gaps states. Their detailed knowledge seems thus to be a timely and important issue.

It is our intention here to gather a systematic information on the in-gap Andreev states originating from the scattering either on the magnetic or non-magnetic quantum impurities. Physical aspects of such study have been so far addressed by a number of groups using various techniques [6]. Since this problem is presently important [11, 12] we would like to collect the essential results into this single report. Subgap states of the magnetic (Kondo-type) impurities have been extensively investigated, both theoretically [13] and experimentally using the two-terminal [14] as well as three-terminal configurations [23]. We would like to emphasize, however, that in-gap states are present also in the case of uncorrelated (spinless) quantum dots [15-17]. To illustrate such possibility in section III we briefly analyze the noninteracting quantum dot, considering evolution of the Andreev bound states with respect to $\Delta / \Gamma_{S}$ [where $\Gamma_{\beta}$ denotes the coupling to $\beta=N, S$ lead] for several asymmetric coupling ratios $\Gamma_{S} / \Gamma_{N}$. Next, in sections IV and V, we address the correlation effects responsible for the Coulomb blockade and the Kondo effect.

Our study can be regarded as complementary to the previous pedagogical analysis by J. Bauer et al [15] who focused on the in-gap states of the quantum impurity immersed in a superconducting medium for the limit $\Gamma_{S} \gg \Delta$. We hope that this analysis would be useful for the tunneling spectroscopy using the quantum dots asymmetrically coupled between the superconducting and normal leads in the two- and multi-terminal configurations.

\section{ANDERSON IMPURITY MODEL}

For description of the quantum dot coupled between the normal $(\mathrm{N})$ and superconducting $(\mathrm{S})$ electrodes we use the Anderson impurity model

$$
\begin{aligned}
\hat{H} & =\hat{H}_{N}+\hat{H}_{S}+\sum_{\sigma} \epsilon_{d} \hat{d}_{\sigma}^{\dagger} \hat{d}_{\sigma}+U_{d} \hat{n}_{d \uparrow} \hat{n}_{d \downarrow} \\
& +\sum_{\mathbf{k}, \sigma} \sum_{\beta=N, S}\left(V_{\mathbf{k} \beta} \hat{d}_{\sigma}^{\dagger} \hat{c}_{\mathbf{k} \sigma \beta}+V_{\mathbf{k} \beta}^{*} \hat{c}_{\mathbf{k} \sigma, \beta}^{\dagger} \hat{d}_{\sigma}\right) .
\end{aligned}
$$

Operators $d_{\sigma}\left(d_{\sigma}^{\dagger}\right)$ denote annihilation (creation) of QD electron with spin $\sigma$ and energy level $\varepsilon_{d}$ and $U_{d}$ is the on-dot repulsion (or charging) energy. The last term in (1) represents a hybridization of the QD with the external leads, where the normal electrode is described by the Fermi gas $\hat{H}_{N}=$ $\sum_{\mathbf{k}, \sigma} \xi_{\mathbf{k} N} \hat{c}_{\mathbf{k} \sigma N}^{\dagger} \hat{c}_{\mathbf{k} \sigma N}$ and the superconducting one is takes the conventional BCS form $\hat{H}_{S}=\sum_{\mathbf{k}, \sigma} \xi_{\mathbf{k} S} \hat{c}_{\mathbf{k} \sigma S}^{\dagger} \hat{c}_{\mathbf{k} \sigma S}-$ $\sum_{\mathbf{k}} \Delta\left(\hat{c}_{\mathbf{k} \uparrow S}^{\dagger} \hat{c}_{-\mathbf{k} \downarrow S}^{\dagger}+\hat{c}_{-\mathbf{k} \downarrow S} \hat{c}_{\mathbf{k} \uparrow S}\right)$. The energies $\xi_{\mathbf{k} \beta}=$ $\varepsilon_{\mathbf{k} \beta}-\mu_{\beta}$ are measured with respect to the chemical potentials $\mu_{\beta}$, which can be detuned by the external voltage $\mu_{N}=\mu_{S}+e V$. We shall focus on the low energy features, assuming the wide band limit approximation $\left|V_{\mathbf{k} \beta}\right| \ll D$ (where $-D \leq \varepsilon_{\mathbf{k} \beta} \leq D$ ) and use the coupling constants $\Gamma_{\beta}=2 \pi \sum_{\mathbf{k}, \beta}\left|V_{\mathbf{k} \beta}\right|^{2} \delta\left(\omega-\xi_{\mathbf{k} \beta}\right)$ as useful energy units.

To consider the proximity effect we introduce the matrix Green's function $\boldsymbol{G}_{d}\left(\tau, \tau^{\prime}\right)=\left\langle\left\langle\hat{\Psi}_{d}(\tau) ; \hat{\Psi}_{d}^{\dagger}\left(\tau^{\prime}\right)\right\rangle\right\rangle$ in the Nambu representation $\hat{\Psi}_{d}^{\dagger}=\left(\hat{d}_{\uparrow}^{\dagger}, \hat{d}_{\downarrow}\right), \hat{\Psi}_{d}=\left(\hat{\Psi}_{d}^{\dagger}\right)^{\dagger}$. Under equilibrium conditions the Green's function $\boldsymbol{G}_{d}\left(\tau, \tau^{\prime}\right)$ depends only on a time difference $\tau-\tau^{\prime}$. Its Fourier transform obeys the following Dyson equation

$$
\boldsymbol{G}_{d}(\omega)^{-1}=\left(\begin{array}{cc}
\omega-\varepsilon_{d} & 0 \\
0 & \omega+\varepsilon_{d}
\end{array}\right)-\boldsymbol{\Sigma}_{d}^{0}(\omega)-\boldsymbol{\Sigma}_{d}^{U}(\omega)
$$

where the selfenergy $\boldsymbol{\Sigma}_{d}^{0}$ corresponds to the noninteracting case $(U=0)$ and the second contribution $\boldsymbol{\Sigma}_{d}^{U}$ refers to the correlation effects induced by local Coulomb repulsion $U_{d} \hat{n}_{d, \uparrow} \hat{n}_{d, \downarrow}$. The uncorrelated quantum dot is characterized by

$$
\boldsymbol{\Sigma}_{d}^{0}(\omega)=\sum_{\mathbf{k}, \beta}\left|V_{\mathbf{k} \beta}\right|^{2} \boldsymbol{g}_{\beta}(\mathbf{k}, \omega),
$$

where $\boldsymbol{g}_{N}(\mathbf{k}, \omega)$ is the Green's function of the normal lead

$$
\boldsymbol{g}_{N}(\mathbf{k}, \omega)=\left(\begin{array}{cc}
\frac{1}{\omega-\xi_{\mathbf{k} N}} & 0 \\
0 & \frac{1}{\omega+\xi_{\mathbf{k} N}}
\end{array}\right)
$$

and $\boldsymbol{g}_{S}(\mathbf{k}, \omega)$ denotes the Green's function of superconducting electrode

$$
\boldsymbol{g}_{S}(\mathbf{k}, \omega)=\left(\begin{array}{cc}
\frac{u_{\mathbf{k}}^{2}}{\omega-E_{\mathbf{k}}}+\frac{v_{\mathrm{k}}^{2}}{\omega+E_{\mathbf{k}}} & \frac{-u_{\mathbf{k}} v_{\mathbf{k}}}{\omega-E_{\mathbf{k}}}+\frac{u_{\mathbf{k}} v_{\mathbf{k}}}{\omega+E_{\mathbf{k}}} \\
\frac{-u_{\mathbf{k}} v_{\mathbf{k}}}{\omega-E_{\mathbf{k}}}+\frac{u_{\mathbf{k}} v_{\mathbf{k}}}{\omega+E_{\mathbf{k}}} & \frac{u_{\mathbf{k}}^{2}}{\omega+E_{\mathbf{k}}}+\frac{v_{\mathbf{k}}^{2}}{\omega-E_{\mathbf{k}}}
\end{array}\right) .
$$

The quasiparticle energies are given by $E_{\mathbf{k}}=\sqrt{\xi_{\mathbf{k} S}^{2}+\Delta^{2}}$ and the usual BCS coefficients take a form $u_{\mathbf{k}}^{2}, v_{\mathbf{k}}^{2}=$ $\frac{1}{2}\left[1 \pm \frac{\xi_{\mathbf{k} S}}{E_{\mathbf{k}}}\right], u_{\mathbf{k}} v_{\mathbf{k}}=\frac{\Delta}{2 E_{\mathbf{k}}}$. In the wide-band limit the selfenergy (3) simplifies to

$$
\Sigma_{d}^{0}(\omega)=-i \frac{\Gamma_{N}}{2}\left(\begin{array}{ll}
1 & 0 \\
0 & 1
\end{array}\right)-\frac{\Gamma_{S}}{2} \gamma(\omega)\left(\begin{array}{cc}
1 & \frac{\Delta}{\omega} \\
\frac{\Delta}{\omega} & 1
\end{array}\right)
$$

with $\omega$-dependent function

$$
\gamma(\omega)= \begin{cases}\frac{\omega}{\sqrt{\Delta^{2}-\omega^{2}}} \text { for }|\omega|<\Delta, \\ \frac{i|\omega|}{\sqrt{\omega^{2}-\Delta^{2}}} \text { for }|\omega|>\Delta .\end{cases}
$$

For considering the correlation effects $\Sigma_{d}^{U}(\omega)$ one has to introduce some approximations. We shall come back to this non-trivial problem in sections IV and V.

\section{IN-GAP STATES OF THE UNCORRELATED QUANTUM DOT}

Let us start by considering the uncorrelated QD, which is equivalent to the spinless impurity. We discuss here the spectroscopic properties of such QD for an arbitrary ratio $\Delta / \Gamma_{S}$ and asymmetric couplings $\Gamma_{N} \neq \Gamma_{S}$. In-gap 

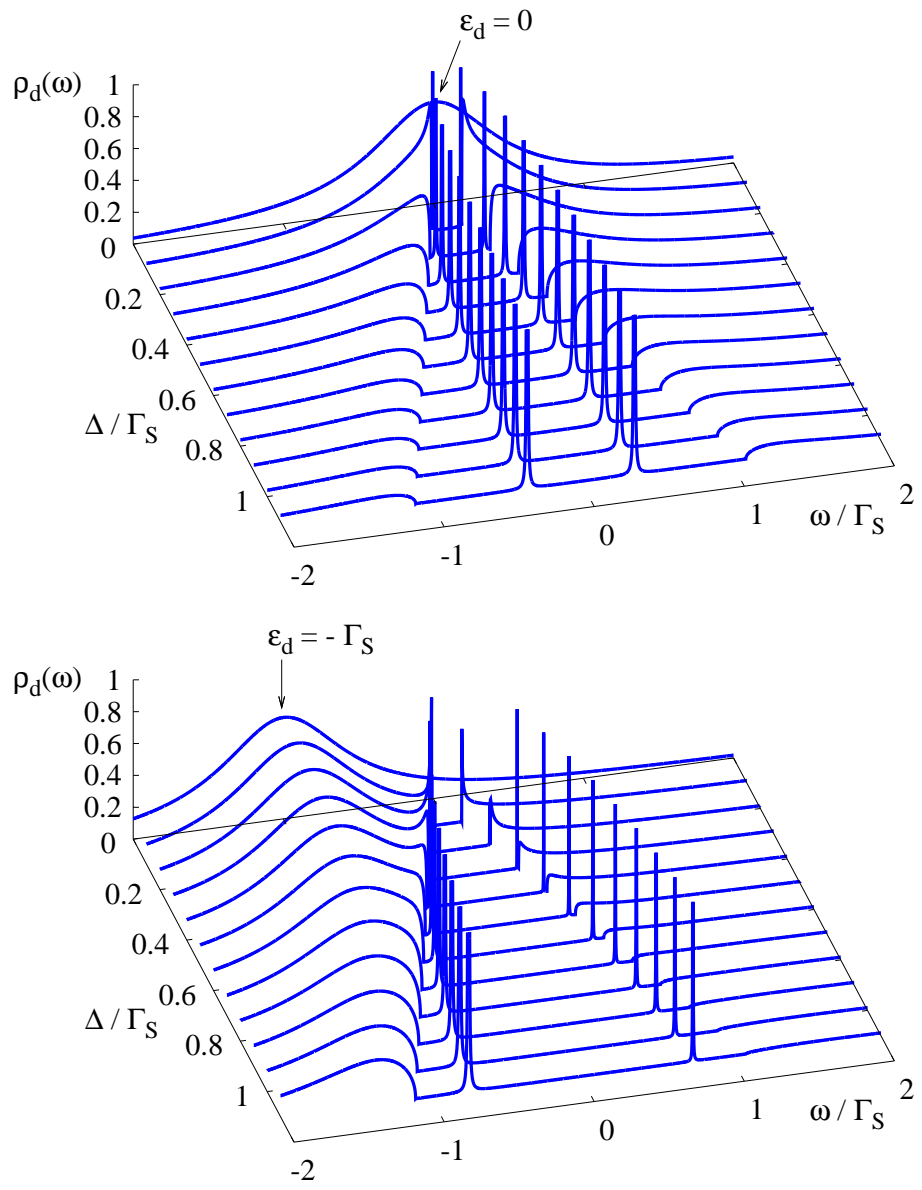

FIG. 1: (color online) Spectral function $\rho_{d}(\omega)$ of the uncorrelated quantum dot obtained for $\varepsilon_{d}=0$ (upper panel) and $\varepsilon_{d}=-\Gamma_{S}$ (bottom panel) assuming weak coupling to the metallic lead $\Gamma_{N}=0.001 \Gamma_{S}$. In both cases the in-gap states gradually emerge from the gap edge singularities $\pm \Delta$ (when $\left.\Delta \ll \Gamma_{S}\right)$ and they evolve to the well-defined subgap quasiparticle peaks (when $\Delta \gg \Gamma_{S}$ ).

states formally represent the poles of the matrix Green's function $\boldsymbol{G}_{d}(\omega)$ existing in the subgap regime $|\omega|<\Delta$. For the uncorrelated quantum $\operatorname{dot}\left(U_{d} \rightarrow 0\right)$ one has

$$
\begin{aligned}
\boldsymbol{G}_{d}(\omega) & =\frac{1}{\left(\tilde{\omega}+i \frac{\Gamma_{N}}{2}\right)^{2}-\varepsilon_{d}^{2}-\left(\frac{\tilde{\Gamma}_{S}}{2}\right)^{2}} \\
& \times\left(\begin{array}{cc}
\tilde{\omega}+i \frac{\Gamma_{N}}{2}+\varepsilon_{d} & -\frac{\tilde{\Gamma}_{s}}{2} \\
-\frac{\tilde{\Gamma}_{s}}{2} & \tilde{\omega}+i \frac{\Gamma_{N}}{2}-\varepsilon_{d}
\end{array}\right)
\end{aligned}
$$

with the following meaning of the symbols $\tilde{\omega}$ and $\tilde{\Gamma}_{S}$

$$
\begin{aligned}
\tilde{\omega} & =\omega+\frac{\Gamma_{S}}{2} \frac{\omega}{\sqrt{\Delta^{2}-\omega^{2}}}, \\
\tilde{\Gamma}_{s} & =\Gamma_{S} \frac{\Delta}{\sqrt{\Delta^{2}-\omega^{2}}} .
\end{aligned}
$$

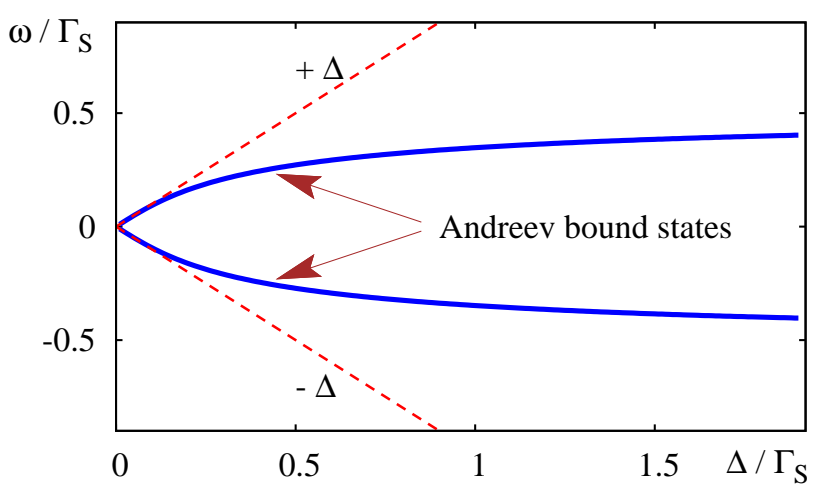

FIG. 2: (color online) Energies of the in-gap states versus the ratio $\Delta / \Gamma_{S}$ obtained for the uncorrelated quantum dot $\left(\varepsilon_{d}=0\right)$ weakly coupled to the metallic lead $\Gamma_{N}=0.001 \Gamma_{S}$. Dashed lines indicate the gap edges $\pm \Delta$.

In this case the single-particle spectral function $\rho_{d}(\omega) \equiv$ - $\frac{1}{\pi} \operatorname{Im}\left\{\boldsymbol{G}_{d, 11}(\omega)\right\}$ is expressed by the standard BCS form

$$
\begin{aligned}
\boldsymbol{G}_{d, 11}(\omega) & =\frac{1}{2}\left[1+\frac{\varepsilon_{d}}{\tilde{E}_{d}}\right] \frac{1}{\tilde{\omega}+i \frac{\Gamma_{N}}{2}-\tilde{E}_{d}} \\
& +\frac{1}{2}\left[1-\frac{\varepsilon_{d}}{\tilde{E}_{d}}\right] \frac{1}{\tilde{\omega}+i \frac{\Gamma_{N}}{2}+\tilde{E}_{d}}
\end{aligned}
$$

with $\omega$-dependent parameter

$$
\tilde{E}_{d}=\sqrt{\varepsilon_{d}^{2}+\left(\tilde{\Gamma}_{s} / 2\right)^{2}} .
$$

In figure 1 we show the spectrum $\rho_{d}(\omega)$ as a function of the energy gap $\Delta$ obtained for the uncorrelated (spinless) quantum dot with $\varepsilon_{d}=0$ and $\varepsilon_{d}=-\Gamma_{S}$. We have assumed a weak coupling to the normal lead $\Gamma_{N} \ll \Gamma_{S}$ what yields a nearly resonant character of the in-gap states. For larger $\Gamma_{N}$ the in-gap states broadening increases (lifetime decreases). We furthermore notice (see Fig. 2), that the Andreev states appear near the gap edge singularities (for $\Delta \ll \Gamma_{S}$ ) and they evolve into the subgap peaks centered at energies $\pm \sqrt{\varepsilon_{d}^{2}+\Gamma_{S}^{2} / 4}$ (for $\Delta \gg \Gamma_{S}$ ).

\section{A. Resonances in the weak coupling limit $\Gamma_{N} \rightarrow 0$}

To get some correspondence with the previous study 15] we now consider in more detail the case of infinitesimally weak coupling to the normal electrode $\Gamma_{N} \rightarrow 0^{+}$. Under such condition the in-gap states become strictly resonant, i.e. they represent the quasiparticles of an infinite life-time. In the subgap regime the Eqn (12) yields the following spectral function

$$
\begin{aligned}
\lim _{|\omega|<\Delta} \rho_{d}(\omega) & =\frac{1}{2}\left(1+\frac{\varepsilon_{d}}{\tilde{E}_{d}}\right) \delta\left[\tilde{\omega}-\tilde{E}_{d}\right] \\
& +\frac{1}{2}\left(1-\frac{\varepsilon_{d}}{\tilde{E}_{d}}\right) \delta\left[\tilde{\omega}+\tilde{E}_{d}\right] .
\end{aligned}
$$


This function (13) can be rewritten as

$$
\lim _{|\omega|<\Delta} \rho_{d}(\omega)=\mathcal{W}_{+} \delta\left[\omega-E_{+}\right]+\mathcal{W}_{-} \delta\left[\omega-E_{-}\right]
$$

with the quasiparticle energies $E_{ \pm}$representing the solutions of the following equation

$$
E_{ \pm}+\frac{\Gamma_{S}}{2} \frac{E_{ \pm}}{\sqrt{\Delta^{2}-E_{ \pm}^{2}}}= \pm \sqrt{\varepsilon_{d}^{2}+\left(\frac{\Gamma_{S}}{2}\right)^{2} \frac{\Delta^{2}}{\Delta^{2}-E_{ \pm}^{2}}} .
$$

and $\mathcal{W}_{ \pm}$being their spectral weights.

We illustrate in figure 2 the energies $E_{ \pm}$of the in-gap resonances versus the ratio $\Delta / \Gamma_{S}$ obtained for $\varepsilon_{d}=0$. In the case of small energy gap $\Delta \ll \Gamma_{S}$ (studied by J. Bauer et al [15]) the resonant in-gap states are located nearby the gap edge singularities $\pm \Delta$. For increasing $\Delta / \Gamma_{S}$ they gradually move aside from the gap edge singularities, and in the limit $\Delta \gg \Gamma_{S}$ approach the asymptotic values $\pm \sqrt{\varepsilon_{d}+\left(\Gamma_{S} / 2\right)^{2}}$. In the next section we discuss in some more detail this 'superconducting atomic' limit $\Delta \gg \Gamma_{S}$.

\section{B. Superconducting atomic limit $\Delta \gg \Gamma_{S}$}

Deep inside the energy gap (i.e. for $|\omega| \ll \Delta$ ) all electronic states of the uncorrelated quantum dot can be determined analytically (for arbitrary $\Gamma_{\beta}$ ) due to the fact, that the selfenergy (6) simplifies then to a static value

$$
\boldsymbol{\Sigma}_{d}^{0}(\omega)=-\frac{1}{2}\left(\begin{array}{cc}
i \Gamma_{N} & \Gamma_{S} \\
\Gamma_{S} & i \Gamma_{N}
\end{array}\right)
$$

Under such conditions the quantum dot can be regarded as the 'superconducting island' with the induced pairing gap $\Delta_{d}=\left|\Gamma_{S} / 2\right|$. This problem has been widely discussed in the literature adopting various methods to describe the correlation effects $U_{d}$ (see sections IV \& V).

The spectral function $\rho_{d}(\omega)$ of the uncorrelated $\mathrm{QD}$ can be expressed explicitly by

$$
\begin{aligned}
\rho_{d}(\omega) & =\frac{1}{2}\left[1+\frac{\varepsilon_{d}}{E_{d}}\right] \frac{\frac{1}{\pi} \Gamma_{N} / 2}{\left(\omega-E_{d}\right)^{2}+\left(\Gamma_{N} / 2\right)^{2}} \\
& +\frac{1}{2}\left[1-\frac{\varepsilon_{d}}{E_{d}}\right] \frac{\frac{1}{\pi} \Gamma_{N} / 2}{\left(\omega+E_{d}\right)^{2}+\left(\Gamma_{N} / 2\right)^{2}}
\end{aligned}
$$

with the quasiparticle energy $E_{d}=\sqrt{\varepsilon_{d}^{2}+\Delta_{d}^{2}}$. The subgap spectrum consists thus of the particle and hole peaks at $\omega= \pm E_{d}$ whose spectral weights depend on $\varepsilon_{d}$ and a broadening is controlled by $\Gamma_{N}$. These particle and hole Lorentzians are well separated from each other until $\Gamma_{S} \geq \Gamma_{N}$. Otherwise they merge into a single structure (see the second reference of [38]).

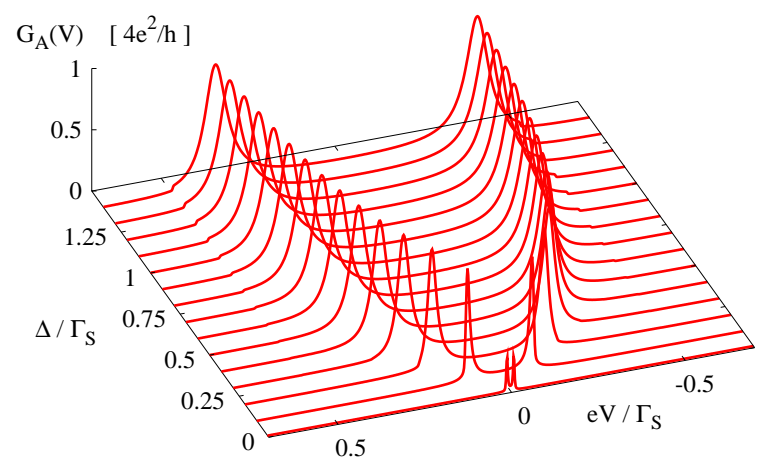

FIG. 3: (color online) The subgap Andreev conductance obtained for the uncorrelated quantum dot with $\varepsilon_{d}=0$ and asymmetric couplings $\Gamma_{N} / \Gamma_{S}=0.1$.

\section{Tunneling spectroscopy}

Any experimental verification of the subgap states is possible only indirectly, by measuring the differential conductance of the tunneling current $I(V)$. In general the charge transport induced through the N-QD-S junction consists of the quasiparticle (QP) and Andreev (A) currents $I(V)=I_{Q P}(V)+I_{A}(V)$. They can expressed in the Landauer-type form [32]

$$
\begin{aligned}
I_{Q P}(V) & =\frac{2 e}{h} \int d \omega T_{Q P}(\omega)[f(\omega-e V)-f(\omega)] \\
I_{A}(V) & =\frac{2 e}{h} \int d \omega T_{A}(\omega)[f(\omega-e V)-f(\omega+e V 0] 9)
\end{aligned}
$$

with the Fermi distribution $f(\omega)=\left[\exp \left(\omega / k_{B} T\right)+1\right]^{-1}$. Transmittance of the Andreev channel $T_{A}(\omega)$ depends on the off-diagonal part of the Green's function

$$
T_{A}(\omega)=\left|\Gamma_{N}\right|^{2}\left|\boldsymbol{G}_{d, 12}(\omega)\right|^{2}
$$

whereas the effective quasiparticle transmittance $T_{Q P}(\omega)$ contains several contributions

$$
\begin{aligned}
T_{Q P}(\omega) & =\Gamma_{N} \Gamma_{S}\left(\left|\boldsymbol{G}_{d, 11}(\omega)\right|^{2}+\left|\boldsymbol{G}_{d, 12}(\omega)\right|^{2}\right. \\
& \left.-\frac{\Delta}{\omega} \operatorname{Re}\left\{\boldsymbol{G}_{d, 11}(\omega) \boldsymbol{G}_{d, 12}^{*}(\omega)\right\}\right) .
\end{aligned}
$$

Usually the off-diagonal Green's function $\boldsymbol{G}_{d, 12}(\omega)$ quickly vanishes outside the energy gap, therefore for $|\omega| \geq \Delta$ the tunneling current simplifies to the popular Meir-Weingreen formula

$$
\begin{aligned}
\lim _{|e V| \geq \Delta} I(V) & \approx \frac{2 e}{h} \int d \omega \Gamma_{N} \Gamma_{S}\left|\boldsymbol{G}_{d, 11}(\omega)\right|^{2} \\
& \times[f(\omega-e V)-f(\omega)] .
\end{aligned}
$$

In the subgap regime $|\omega|<\Delta_{d}$ (especially for the strongly asymmetric couplings $\Gamma_{S} \gg \Gamma_{N}$ ) the transport is solely 


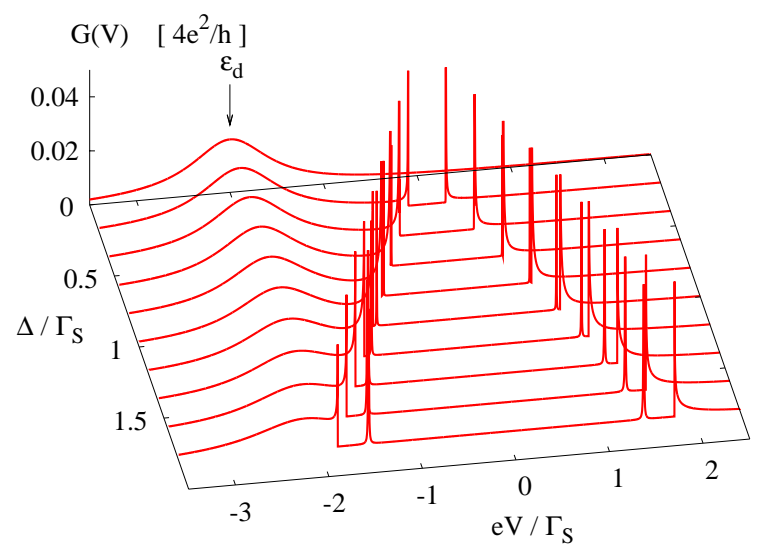

FIG. 4: (color online) The effective differential conductance of the uncorrelated quantum dot asymmetrically coupled to the external leads $\Gamma_{N} / \Gamma_{S}=0.01$ obtained for $\varepsilon_{d}=-2 \Gamma_{S}$.

provided by the Andreev current (19). Figure 3 shows the Andreev conductance $G_{A}(V)=\frac{d}{d V} I_{A}(V)$ obtained for $\Gamma_{N}=0.1 \Gamma_{S}$. We can notice that the differential conductance is similar (although not identical) with the ingap spectrum $\rho_{d}(\omega)$ presented in figures 1 and 2 .

The next plot 4 illustrates the total conductance $G(V)=\frac{d}{d V} I(V)$ obtained at $T=0$ for $\varepsilon_{d}=-2 \Gamma_{S}$. In these curves we can clearly identify: a) the broad peak at $\left.e V=\varepsilon_{d}, \mathrm{~b}\right)$ the signatures of gap edge singularities (manifested by sharp enhancement of the single particle tunneling at $e V= \pm \Delta$ ), and c) the well pronounced ingap features related to the Andreev bound states. For $\Delta>\Gamma_{S}$ the in-gap features are well separated from the gap edge singularities, otherwise it is rather difficult to recognize them for the coupling $\Gamma_{N} \geq \Gamma_{S}$ (for instance, see figure 2 in the Ref. [13]). The experimental data of Deacon et al [8] clearly confirmed such well defined subgap peaks in the Andreev conductance for the strongly asymmetric coupling $\Gamma_{S} \geq 40 \Gamma_{N}$.

\section{CORRELATION EFFECTS}

Interplay between the Coulomb repulsion and the induced on-dot pairing is, in general, a very complicated issue. To gain some insight regarding their competition we shall consider the strongly asymmetric limit $\Gamma_{S} \gg \Gamma_{N}$ (i.e. assuming $\Gamma_{N}=0^{+}$). In absence of the Coulomb repulsion the strong hybridization to superconducting electrode converts the quantum dot into 'superconducting impurity' with the induced pairing gap $\Delta_{d}=\Gamma_{S} / 2$. Influence of the Coulomb repulsion $U_{d}$ on the subgap Andreev states in the large gap limit with a vanishing coupling to the normal lead has been first addressed by E. Vecino et al 24]. Comparison of the methods used for determination of the bound states of the 'superconduct- ing Anderson model' have been recently revisited in Ref. [25]. In what follows below, we briefly summarize the essential results based on the exact solution of the effective 'superconducting' QD Hamiltonian [26]

$$
\hat{H}_{Q D}=\sum_{\sigma} \varepsilon_{d} \hat{d}_{\sigma}^{\dagger} \hat{d}_{\sigma}-\Delta_{d}\left(\hat{d}_{\uparrow}^{\dagger} \hat{d}_{\downarrow}^{\dagger}+\hat{d}_{\downarrow} \hat{d}_{\uparrow}\right)+U \hat{n}_{d \downarrow} \hat{n}_{d \uparrow},(23)
$$

where the proximity effect is taken into account by the pair source/sink terms. The doublet configurations $|\uparrow\rangle$ and $|\downarrow\rangle$ (corresponding to total spin $S=\frac{1}{2}$ ) represent true eigenstates with the eigenvalue $\varepsilon_{d}$. The other singlet states $(S=0)$ can be expressed as linear combinations of the empty and doubly occupied sites

$$
\begin{aligned}
& \left|\Psi_{-}\right\rangle=u_{d}|0\rangle-v_{d}|\uparrow \downarrow\rangle, \\
& \left|\Psi_{+}\right\rangle=v_{d}|0\rangle+u_{d}|\uparrow \downarrow\rangle .
\end{aligned}
$$

The corresponding eigenenergies are given by [15, 24, 27]

$$
E_{\mp}=\left(\varepsilon_{d}+\frac{U_{d}}{2}\right) \mp \sqrt{\left(\varepsilon_{d}+\frac{U_{d}}{2}\right)^{2}+\Delta_{d}^{2}}
$$

and the diagonalization coefficients $u_{d}, v_{d}$ take the form

$$
u_{d}^{2}=\frac{1}{2}\left[1+\frac{\varepsilon_{d}+U_{d} / 2}{E_{d}}\right]=1-v_{d}^{2}
$$

with $E_{d}=\sqrt{\left(\varepsilon_{d}+U_{d} / 2\right)^{2}+\Delta_{d}^{2}}$. Using the spectral Lehmann representation we can determine the full matrix Green's function $\boldsymbol{G}_{Q D}(\omega)$ of the 'superconducting atomic limit' (in the case $\Gamma_{N}=0^{+}$). Because of the Coulomb blockade it takes effectively the four-pole structure

$$
\begin{aligned}
\boldsymbol{G}_{Q D, 11}(\omega) & =\frac{\alpha u_{d}^{2}}{\omega-\left(\frac{U_{d}}{2}+E_{d}\right)}+\frac{\beta v_{d}^{2}}{\omega-\left(\frac{U_{d}}{2}-E_{d}\right)} \\
& +\frac{\alpha v_{d}^{2}}{\omega+\left(\frac{U_{d}}{2}+E_{d}\right)}+\frac{\beta u_{d}^{2}}{\omega+\left(\frac{U_{d}}{2}-E_{d}\right)}(28) \\
\boldsymbol{G}_{Q D, 12}(\omega) & =\frac{\alpha u_{d} v_{d}}{\omega-\left(\frac{U_{d}}{2}+E_{d}\right)}-\frac{\beta u_{d} v_{d}}{\omega-\left(\frac{U_{d}}{2}-E_{d}\right)} \\
& -\frac{\alpha u_{d} v_{d}}{\omega+\left(\frac{U_{d}}{2}+E_{d}\right)}+\frac{\beta u_{d} v_{d}}{\omega+\left(\frac{U_{d}}{2}-E_{d}\right)}(29)
\end{aligned}
$$

and $\boldsymbol{G}_{Q D, 22}(\omega)=-\left[\boldsymbol{G}_{Q D, 11}(-\omega)\right]^{*}, \boldsymbol{G}_{Q D, 12}(\omega)=$ $\left[\boldsymbol{G}_{Q D, 21}(-\omega)\right]^{*}$. The relative spectral weights $\alpha$ and $\beta$ are given by

$$
\begin{aligned}
\alpha & =\frac{\exp \left\{\frac{U_{d}}{2 k_{B} T}\right\}+\exp \left\{-\frac{E_{d}}{k_{B} T}\right\}}{2 \exp \left\{\frac{U_{d}}{2 k_{B} T}\right\}+\exp \left\{-\frac{E_{d}}{k_{B} T}\right\}+\exp \left\{\frac{E_{d}}{k_{B} T}\right\}} \\
& =1-\beta .
\end{aligned}
$$

Spectrum of the correlated quantum dot consists of the four in-gap resonances at quasiparticle energies $\pm \frac{U_{d}}{2} \pm$ $E_{d}$. For arbitrary $U_{d}$ the spectral function $\rho_{Q D}(\omega) \equiv$ 
$-\frac{1}{\pi} \operatorname{Im}\left\{\boldsymbol{G}_{Q D, 11}(\omega)\right\}$ takes the following form

$$
\begin{aligned}
& \rho_{Q D}(\omega)= \\
= & \alpha u_{d}^{2} \delta\left(\omega-\frac{U_{d}}{2}-E_{d}\right)+\beta v_{d}^{2} \delta\left(\omega-\frac{U_{d}}{2}+E_{d}\right) \\
+ & \alpha v_{d}^{2} \delta\left(\omega+\frac{U_{d}}{2}+E_{d}\right)+\beta u_{d}^{2} \delta\left(\omega+\frac{U_{d}}{2}-E_{d}\right) .
\end{aligned}
$$

This spectral function (31) obeys the sum rule $\int_{-\infty}^{\infty} \rho_{Q D}(\omega) d \omega=1$. For $U_{d}=0$ it properly reproduces the exact BCS-type solution $\lim _{U_{d} \rightarrow 0} \rho_{Q D}(\omega)=$ $u_{d}^{2} \delta\left(\omega-\sqrt{\varepsilon_{d}^{2}+\Delta_{d}^{2}}\right)+v_{d}^{2} \delta\left(\omega+\sqrt{\varepsilon_{d}^{2}+\Delta_{d}^{2}}\right)$. Using (23) we can discuss the qualitative effects due to a competition between the Coulomb interactions and the proximity induced on-dot pairing. This aspect has been practically investigated in various nanoscopic setups [8, 11, 12]. Expansions around this 'superconducting atomic limit' (for $\Gamma_{N} \neq 0$ ) have been developed in the Refs 28,29$]$.

Since the most profound influence of the Coulomb repulsion takes place in the particle-hole symmetric case (i.e. for $\varepsilon_{d}=-U_{d} / 2$ ) we shall explore this situation, focusing on the fate in-gap resonances upon varying $U_{d}$. The Coulomb potential $U_{d}$ directly affects the quasiparticle energies $\pm U_{d} / 2 \pm \Delta_{d}$ and their spectral weights. By inspecting (26) we can notice, that for $\Delta_{d}=\frac{1}{2} U_{d}$ the ground state evolves from the BCS singlet to the doublet configuration [15]. This crossover is accompanied by significant redistribution of the spectral weights

$$
\lim _{T \rightarrow 0} \alpha= \begin{cases}0 & \text { for } \frac{1}{2} U_{d}<\Delta, \\ \frac{1}{3} & \text { for } \frac{1}{2} U_{d}=\Delta, \\ \frac{1}{2} & \text { for } \frac{1}{2} U_{d}>\Delta .\end{cases}
$$

In the weak interaction limit $\frac{1}{2} U_{d}<\Delta$ (corresponding to the BCS singlet state) the spectrum consists of two peaks

$\rho_{Q D}(\omega)=\frac{1}{2} \delta\left(\omega-\frac{U_{d}}{2}+\Delta_{d}\right)+\frac{1}{2} \delta\left(\omega+\frac{U_{d}}{2}-\Delta_{d}\right)$,

separated by an effective energy gap $2 \Delta_{d}-U_{d}$. At the singlet-doublet crossover (i.e. for $\frac{1}{2} U_{d}=\Delta$ ) the spectrum evolves to three-pole structure

$$
\rho_{Q D}(\omega)=\frac{1}{6} \delta\left(\omega+2 \Delta_{d}\right)+\frac{1}{6} \delta\left(\omega-2 \Delta_{d}\right)+\frac{2}{3} \delta(\omega) .
$$

Finally, in the strong interaction limit $\frac{1}{2} U_{d}>\Delta$ (corresponding to the doublet configuration), the spectral function $\rho_{Q D}(\omega)$ consists of the four in-gap resonances

$$
\rho_{Q D}(\omega)=\frac{1}{4} \delta\left(\omega \pm \frac{U_{d}}{2} \pm \Delta_{d}\right)
$$

In the non-symmetric case $\varepsilon_{d} \neq-U_{d} / 2$ the singletdoublet crossover occurs at larger values of $U_{d}$, but still
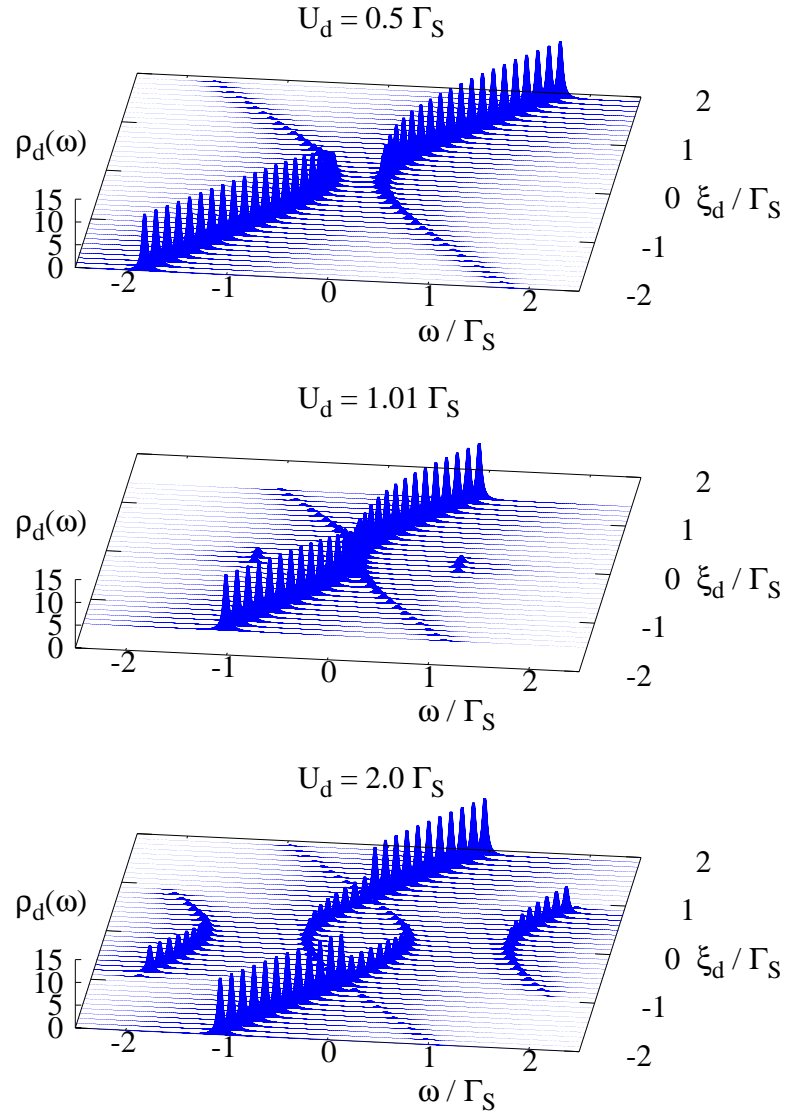

FIG. 5: (color online) Spectral function $\rho_{d}(\omega)$ of the correlated quantum obtained at $T=0$ in the 'superconducting atomic limit' $\Delta \gg \Gamma_{S}$ for several values of the Coulomb potential $U_{d}$. We plot the spectral function with respect to the energy $\xi_{d} \equiv \varepsilon_{d}+\frac{U_{d}}{2}$ for $U_{d} / \Gamma_{S}=0.5$ (upper panel), 1.01 (middle panel) and 2.0 (lower panel).

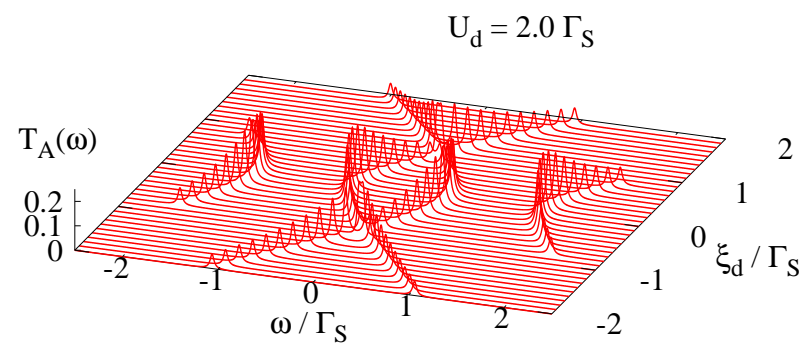

FIG. 6: (color online) Andreev transmittance (20) obtained at $T=0$ in the 'superconducting atomic limit' for $U_{d}=2 \Gamma_{S}$. 
the QP spectrum comprises either two, three, or four subgap Andreev states. We illustrate this behavior in figures 5 and 6, where the coupling $\Gamma_{N}$ to the normal electrode is treated via the simple substitution $\boldsymbol{G}_{d}^{-1}(\omega)=$ $\left[\boldsymbol{G}_{Q D}(\omega)\right]^{-1}+\frac{i}{2} \Gamma_{N} \boldsymbol{I}[27]$. Influence of the metallic lead causes then a broadening of the subgap states.

\section{SUBGAP STATES IN THE KONDO REGIME}

In the last section we address additional effects characteristic for the Kondo regime. This issue has been studied in the literature by a number of authors using a variety of methods, such as: equation of motion technique [30], slave boson approach [31, 32], non-crossing approximation [33], iterated perturbation theory [34, 35], Keldysh Green's function approach combined with the path integral formalism and dynamical mean field approximation [36], numerical renormalization group [26, 37], modified equation of motion approach [38], functional renormalization group [39], cotunneling approach (for the spinful dot) [13], Quantum Monte Carlo simulations [40] and other [41, 42]. In particular, it has been checked whether the Kondo resonance could be somehow manifested in the subgap conductance.

For studying the qualitative features caused by the Kondo effect we shall determine the total selfenergy $\boldsymbol{\Sigma}_{d}(\omega)=\boldsymbol{\Sigma}_{d}^{0}(\omega)+\boldsymbol{\Sigma}_{d}^{U}(\omega)$ in the matrix form

$$
\boldsymbol{\Sigma}_{d}(\omega)=\left(\begin{array}{cc}
\Sigma_{\uparrow}^{\operatorname{diag}}(\omega) & \Sigma^{o f f}(\omega) \\
{\left[\Sigma^{o f f}(-\omega)\right]^{*}-\left[\Sigma_{\downarrow}^{\operatorname{diag}}(-\omega)\right]^{*}}
\end{array}\right)
$$

focusing on the subgap limit $\Delta \gg \Gamma_{S}$. We thus consider the correlated quantum dot with the induced on-dot pairing $\Delta_{d}$ coupled to the metallic lead $\hat{H}=$ $\sum_{\sigma} \varepsilon_{d} \hat{d}_{\sigma}^{\dagger} \hat{d}_{\sigma}+U \hat{n}_{d \downarrow} \hat{n}_{d \uparrow}-\Delta_{d}\left(\hat{d}_{\uparrow}^{\dagger} \hat{d}_{\downarrow}^{\dagger}+\hat{d}_{\downarrow} \hat{d}_{\uparrow}\right)+\hat{H}_{N}+$ $\sum_{\mathbf{k}, \sigma}\left(V_{\mathbf{k} N} \hat{d}_{\sigma}^{\dagger} \hat{c}_{\mathbf{k} \sigma N}+\right.$ h.c. $)$. This simplified problem is not solvable exactly therefore we have to impose some approximations. For this purpose we treat the Coulomb interactions within the selfconsistent scheme based on the equation of motion (EOM) approach [44] extended to the case of the on-dot pairing $\Delta_{d} \neq 0$.

As a starting point we express a diagonal part of the Green's function $\boldsymbol{G}$ by the BCS-type pairing Ansatz [43]

$$
\begin{aligned}
{\left[\boldsymbol{G}_{11}(\omega)\right]^{-1} } & =\omega-\varepsilon_{d}-\Sigma_{\uparrow}^{\text {diag }}(\omega) \\
& -\frac{\Delta_{d}^{2}}{\omega+\varepsilon_{d}+\left[\Sigma_{\downarrow}^{\text {diag }}(-\omega)\right]^{*}} .
\end{aligned}
$$

The hole propagator is related to (37) via $\boldsymbol{G}_{22}(\omega)=$ $-\left[\boldsymbol{G}_{11}(-\omega)\right]^{*}$. Let us notice that in the noninteracting case $\lim _{U_{d} \rightarrow 0} \Sigma_{\sigma}^{\operatorname{diag}}(\omega)=-i \Gamma_{N} / 2$. For arbitrary $U_{d} \neq 0$ we estimate $\Sigma_{\sigma}^{\text {diag }}(\omega)$ using the EOM decoupling procedure [44] (for details see Appendix B in Ref. [45]) but our scheme outlined below can be combined also with other approximations, for instance NCA [33], perturbative expansion [34] etc. The EOM approach yields

$$
\Sigma_{\sigma}^{\operatorname{diag}}(\omega) \simeq U_{d}\left[n_{d, \bar{\sigma}}-\Sigma_{1}(\omega)\right]+\frac{U_{d}\left[n_{d, \bar{\sigma}}-\Sigma_{1}(\omega)\right]\left[\Sigma_{3}(\omega)+U_{d}\left(1-n_{d, \bar{\sigma}}\right)\right]}{\omega-\varepsilon_{d}-\Sigma_{0}(\omega)-\left[\Sigma_{3}(\omega)+U_{d}\left(1-n_{d, \bar{\sigma}}\right)\right]},
$$

where $\Sigma_{0}(\omega)=-\frac{i}{2} \Gamma_{N}$

$$
\Sigma_{\nu}(\omega)=\sum_{\mathbf{k}}\left|V_{\mathbf{k} N}\right|^{2}\left[\frac{1}{\omega-\xi_{\mathbf{k} N}}+\frac{1}{\omega-U_{d}-2 \varepsilon_{d}+\xi_{\mathbf{k} N}}\right] \times \begin{cases}f\left(\xi_{\mathbf{k} N}\right) & \text { for } \nu=1 \\ 1 & \text { for } \nu=3\end{cases}
$$

and $\bar{\uparrow}=\downarrow, \bar{\downarrow}=\uparrow$. To determine the off-diagonal parts of $\boldsymbol{G}(\omega)$ we next use the following exact relation

$$
\left(\omega-\varepsilon_{d}\right) \boldsymbol{G}_{11}(\omega)=1+U_{d}\left\langle\left\langle\hat{d}_{\uparrow} \hat{n}_{d \downarrow} ; \hat{d}_{\uparrow}^{\dagger}\right\rangle\right\rangle-\Delta_{d} \boldsymbol{G}_{12}(\omega)(40)
$$

and $\boldsymbol{G}_{21}(\omega)=\boldsymbol{G}_{12}^{*}(-\omega)$. As an approximation we neglect here an influence of the induced on-dot pairing on the two-body propagator $\left\langle\left\langle\hat{d}_{\uparrow} \hat{n}_{d \downarrow} ; \hat{d}_{\uparrow}^{\dagger}\right\rangle\right\rangle$ appearing in (40). This assumption should be justified as long as $U_{d}$ is safely larger than $\Delta_{d}=\Gamma_{S} / 2$. We thus take

$$
\left\langle\left\langle\hat{d}_{\uparrow} \hat{n}_{d \downarrow} ; \hat{d}_{\uparrow}^{\dagger}\right\rangle\right\rangle \simeq \frac{n_{d \downarrow}-\Sigma_{1}(\omega) \boldsymbol{G}_{11}(\omega)}{\omega-\varepsilon_{d}-\Sigma_{0}(\omega)-U_{d}-\Sigma_{3}(\omega)}
$$

which formally originates from the EOM solution [44].

Having this first guess for the matrix Green's function $\boldsymbol{G}(\omega)$ [expressed through the equations (37-41)] we now construct its selfconsistent improvement. We update the initial pairing Ansatz (37) by iteratively substituting the former selfenergy functional $\boldsymbol{\Sigma}[\boldsymbol{G}(\omega)]$ to the true relation

$$
\begin{aligned}
{\left[\boldsymbol{G}_{11}(\omega)\right]^{-1} } & =\omega-\varepsilon_{d}-\Sigma^{\operatorname{diag}}(\omega) \\
& -\frac{\left[\boldsymbol{\Sigma}^{\text {off }}(-\omega)\right]^{*} \boldsymbol{\Sigma}^{o f f}(\omega)}{\omega+\varepsilon_{d}+\left[\Sigma^{\operatorname{diag}}(-\omega)\right]^{*}} .
\end{aligned}
$$

At each step we determine the off-diagonal terms via (40) and continue until a satisfactory convergence is 


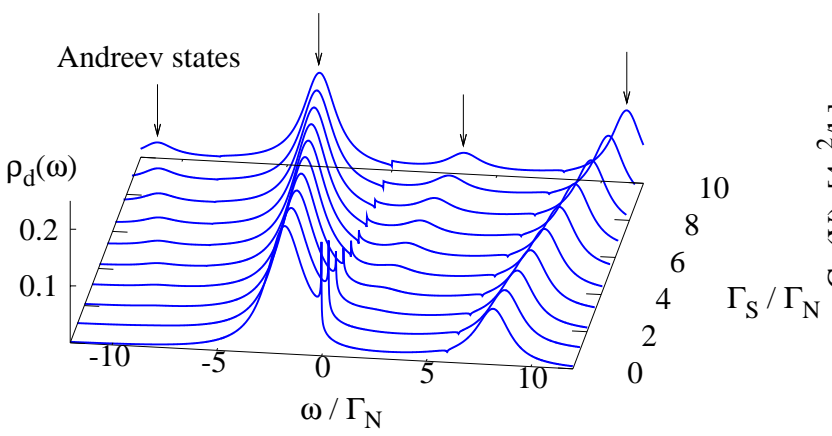

FIG. 7: (color online) Spectral function $\rho_{d}(\omega)$ of the strongly correlated quantum dot obtained in the Kondo regime for $\varepsilon_{d}=-2 \Gamma_{N}, U_{d}=10 \Gamma_{N}$ at temperature $T=0.001 \Gamma_{N} k_{B}^{-1}$ (well below $T_{K}$ ). Calculations have been done assuming $\Delta$ much larger than $U_{d}$.

reached. We have done numerical calculations of the matrix Green's function $\boldsymbol{G}(\omega)$ within such algorithm using the mash of 9000 equidistant energies $\omega_{n}$ slightly above the real axis. We have noticed that practically 7 to 11 iterations were sufficient for a good convergence.

Let us remark that upon neglecting the terms $\Sigma_{1}(\omega)$ and $\Sigma_{3}(\omega)$ of the diagonal selfenergy (38) in the initial iterative step we would recover the usual second order perturbation formula $\lim _{\Sigma_{1}, \Sigma_{3} \rightarrow 0} \Sigma_{\sigma}^{\operatorname{diag}}(\omega)=U_{d} n_{d, \bar{\sigma}}+$ $U_{d}^{2} \frac{n_{d, \bar{\sigma}}\left(1-n_{d, \bar{\sigma}}\right)}{\omega+i \Gamma_{N} / 2-\varepsilon_{d}-U_{d}\left(1-n_{d, \bar{\sigma}}\right)}$. This fact indicates that such simplified selfenergy is able to account for the charging effect (i.e. the Coulomb blockade) discussed by us in the preceding section. In our numerical treatment we keep, however, all the contributions entering (38) because they are important in the Kondo regime $\varepsilon_{d}<0<\varepsilon_{d}+U_{d}$. At temperatures below $k_{B} T_{K}=$ $0.5 \sqrt{U_{d} \Gamma_{N}} \exp \left\{-\pi \frac{\left|\varepsilon_{d}\left(\varepsilon_{d}+U_{d}\right)\right|}{U_{d} \Gamma_{N}}\right\}$ the diverging real part of $\Sigma_{1}(\omega)$ induces then a narrow Abrikosov-Suhl (or Kondo) peak at $\mu_{N}$. From more sophisticated treatments it is known that at low temperatures its broadening should scale with $k_{B} T_{K}$. Unfortunately the EOM approach does not reproduce the low energy structure of the Kondo peak. This missing information could be obtained e.g. from the renormalization group calculations [26, 37], but we nevertheless hope that the overall spectrum and the transport properties are qualitatively properly reproduced by the present treatment.

In figure 7 we show the spectral function of the strongly correlated quantum dot obtained for $\varepsilon_{d}=-2 \Gamma_{N}, U_{d}=$ $10 \Gamma_{N}$ at $k_{B} T=0.0001 \Gamma_{N}$. The curve corresponding to $\Gamma_{S}=0$ (in absence of the proximity effect) reveals the quasiparticle peak at $\varepsilon_{d}$ and its Coulomb satellite at $\varepsilon_{d}+U_{d}$. Both peaks are broadened by $\sim \Gamma_{N}$ (actually, the EOM approximation slightly overestimates such broadening). Besides the quasiparticle in-gap states we also notice the narrow Kondo resonance at $\omega=0$.

For increasing values of the coupling $\Gamma_{S}$ there occurs a gradual formation of the particle and hole in-gap fea-

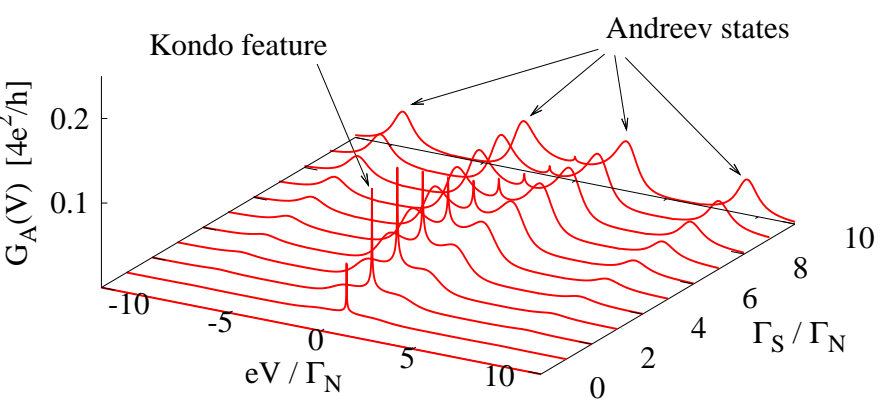

FIG. 8: (color online) The differential Andreev conductance $G_{A}(V)$ of the Kondo regime obtained for the same model parameters as in figure 7 . The zero-bias enhancement (caused by the Abrikosov-Suhl resonance) is gradually suppressed for increasing $\Gamma_{S}$ due to the on-dot pairing.

tures (similar to what has been discussed in the previous sections). This process is accompanied by some qualitative changes of the Kondo resonance. It is gradually suppressed and, for $\Gamma_{S} \geq 4 \Gamma_{N}$ evolves to the kink-type structure characteristic for the mixed valence regime. This behavior is caused by a competition between the on-dot pairing (promoted by $\Gamma_{S}$ ) and the Kondo-type correlations [46]. Signatures of their eventual coexistence occur when $\Gamma_{S}$ is comparable or slightly larger than $\Gamma_{N}$. Under such conditions the subgap spectrum consists of four Andreev quasiparticle peaks (of a broadening $\sim \Gamma_{N}$ ) and the narrow Kondo resonance. These in-gap features can be practically observed by the measurements of the differential conductance in the Kondo regime. The subgap conductance shown in figure 8 reveals all the mentioned peaks of the electronic spectrum (see figure 7) although in a symmetrized way $G_{A}(V)=G_{A}(-V)$ because the particle and hole degrees of freedom equally participate in the Andreev scattering.

\section{CONCLUSIONS}

We have investigated the spectroscopic and transport properties of the quantum impurity coupled to one conducting and another superconducting electrode. The proximity effect depletes electronic states in a subgap region $-\Delta<\omega<\Delta$ but the Andreev-type scattering (i.e. conversion of electrons into the Cooper pairs with a simultaneous reflection of the holes) contributes the ingap states, both in the correlated and the uncorrelated quantum dots. Since the Andreev mechanism involves the particle and hole degrees of freedom there appears an even number of the in-gap bound states.

For a weak coupling to the metallic lead $\Gamma_{N} \ll \Gamma_{S}$ the in-gap states take a form of the narrow resonances, representing the infinite life-time quasiparticles. Otherwise, the in-gap states acquire a finite line-broadening, roughly proportional to $\Gamma_{N}$. The number of in-gap states depends 
sensitively on the Coulomb potential $U_{d}$ (strictly speaking on the ratio $U_{d} / \Delta$ ). For the weak interaction limit the ground state is preferred as the BCS singlet configuration [15, 28, 29], so consequently there appear only two Andreev subgap states. For stronger correlations $U_{d} \gg \Delta$ the number of in-gap states is doubled. Positions of these in-gap states depend on the ratio $\Delta / \Gamma_{S}$. For $\Gamma_{S} \leq \Delta$ the in-gap states are located nearby the gap edge singularities $\sim \pm \Delta$. Otherwise, they move aside from the gap edges and, in the extreme 'superconducting atomic limit' $\Gamma_{S} \gg \Delta$, the subgap quasiparticle energies approach $\pm \frac{1}{2} U_{d} \pm \sqrt{\left(\varepsilon_{d}+U_{d} / 2\right)^{2}+\Gamma_{S}^{2} / 4}$. Coupling to the metallic lead merely affects the line-broadening of such in-gap states.

We have also studied the Kondo regime using iterative scheme based on the equation of motion approximation [44]. In addition to the previously indicated Andreev states we have obtained the narrow Abrikosov-Suhl peak at $\omega=0$. Such Kondo feature is present in the spectrum unless a strong enough coupling to the superconducting lead $\Gamma_{S}$ (promoting the on-dot pairing) gradually suppresses it. In a region where the Kondo state coex- ists with the induced on-dot pairing the spectral function $\rho_{d}(\omega)$ is characterized by five subgap states: four of them represent the Andreev peaks (with a line-broadening $\left.\sim \Gamma_{N}\right)$ and another one is due to the Abrikosov-Suhl resonance (of a broadening $\sim k_{B} T_{K}$ ). These features show up indirectly in the subgap conductance. Signatures of the zero-bias enhancement of the Andreev conductance 5, 8, 23] can be naturally assigned to the Kondo effect. We hope that the present study discussing influence of: (i) the asymmetric couplings $\Gamma_{N} / \Gamma_{S}$, (ii) the energy gap $\Delta / \Gamma_{S}$ and (iii) interplay between the Coulomb repulsion $U_{d}$ and the induced on-dot pairing (promoted by $\Gamma_{S}$ ) would be useful for the experimental studies of the manybody effects in the N-QD-S junctions and in their more complex multi-terminal equivalents [12, 29, 37, 47, 48].

\section{Acknowledgments}

We acknowledge discussions with J. Bauer, S. Andergassen, K.I. Wysokiński and kindly thank for useful comments from R. Aguado and Y. Avishai.
[1] A.V. Balatsky, I. Vekhter, and J.-X. Zhu, Rev. Mod. Phys. 78, 373 (2006).

[2] P.W. Anderson, J. Phys. Chem. Solids 11, 26 (1959).

[3] A.A. Abrikosov and L.P. Gorkov, Sov. Phys. JETP 12, 1243 (1961).

[4] Yu Luh, Acta Phys. Sin. 21, 75 (1965); H. Shiba, Prog. Theor. Phys. 40, 435 (1968); A.I. Rusinov, Sov. Phys. JETP 56, 2047 (1969); H. Shiba and T. Soda, Prog. Theor. Phys. 41, 25 (1969).

[5] E.J.H. Lee, X. Jiang, R. Aguado, G. Katsaros, C.M. Lieber, and S. De Franceschi, Phys. Rev. Lett. 109, 186802 (2012).

[6] A. Martín-Rodero and A. Levy-Yeyati, Adv. Phys. 60, 899 (2011).

[7] S. De Franceschi, L. Kouwenhoven, C. Schönenberger, and W. Wernsdorfer, Nature Nanotechnology 5, 703 (2010).

[8] R.S. Deacon, Y. Tanaka, A. Oiwa, R. Sakano, K. Yoshida, K. Shibata, K. Hirakawa, and S. Tarucha, Phys. Rev. Lett. 104, 076805 (2010); R.S. Deacon, Y. Tanaka, A. Oiwa, R. Sakano, K. Yoshida, K. Shibata, K. Hirakawa, and S. Tarucha, Phys. Rev. B 81, 121308(R) (2010).

[9] J.-D. Pillet, C.H.L. Quay, P. Morfin, C. Bena, A. LevyYeyati, and P. Joyez, Nature Phys. 6, 965 (2010).

[10] V. Mourik, K. Zuo, S.M. Frolov, S.R. Pissard, E.P.A.M. Bakkers, and L.P. Kouwenhoven, Science 336, 1003 (2012).

[11] E.J.H. Lee, X. Jiang, M. Houzet, R. Aguado, Ch.M. Lieber, S. Di Franceschi, arXiv:1302.2611 v2 (preprint).

[12] R. Maurand, Ch. Schönenberger, Physics 6, 75 (2013); J.D. Pillet, P. Joyez, R. Žitko, and F.M. Goffman, Phys. Rev. B 88, 045101 (2013).

[13] V. Koerting, B.M. Andersen, K. Flensberg, and J. Paaske, Phys. Rev. B 82, 245108 (2010); B.M. Ander- sen, K. Flensberg, V. Koerting, and J. Paaske, Phys. Rev. Lett. 107, 256802 (2011).

[14] F. Hübler, M.J. Wolf, T. Scherer, D. Wang, D. Beckmann, and H. v. Löhneysen, Phys. Rev. Lett. 109, 087004 (2012).

[15] J. Bauer, A. Oguri, and A.C. Hewson, J. Phys.: Condens. Matter 19, 486211 (2008).

[16] T. Hecht, A. Weichselbaum, J. von Delft, and R. Bulla, J. Phys.: Condens. Matter 20, 275213 (2008).

[17] C.W.J. Beenakker, Phys. Rev. B 46, 12841 (1992).

[18] L. Hofstetter, S. Csonka, J. Nygård, C. Schönenberger, Nature 461, 960 (2009).

[19] L.G. Herrmann, F. Portier, P. Roche, A. Levy Yeyati, T. Kontos, C. Strunk, Phys. Rev. Lett. 104, 026801 (2010).

[20] J. Schindele, A. Baumgartner, and C. Schönenberger, Phys. Rev. Lett. 109, 157002 (2012).

[21] L. Hofstetter, A. Geresdi, M. Aagesen, J. Nygård, C. Schönenberger, and S. Csonka, Phys. Rev. Lett. 104, 246804 (2010).

[22] B.-K. Kim, Y.-H. Ahn, J.-J. Kim, M.-S Choi, M.-H. Bae, K. Kang, J.S. Lim, R. Lopez, and N. Kim, Phys. Rev. Lett. 110, 076803 (2013).

[23] W. Chang, V.E. Manucharyan, T.S. Jaspersen, J. Nygård, and C.M. Marcus, Phys. Rev. Lett. 110, 217005 (2013).

[24] E. Vecino, A. Martín-Rodero, and A. Levy Yeyati, Phys. Rev. B 68, 035105 (2003).

[25] A. Martín-Rodero and A. Levy Yeyati, J. Phys.: Condens. Matter 24, 385303 (2012).

[26] Y. Tanaka, N. Kawakami, and A. Oguri, J. Phys. Soc. Jpn. 76, 074701 (2007); Y. Yamada, Y. Tanaka, and N. Kawakami, Phys. Rev. B 84, 075484 (2011).

[27] K.I. Wysokiński, J. Phys.: Condens. Matter 24, 335303 (2012).

[28] T. Meng, S. Florens, and P. Simon, Phys. Rev. B 79, 
224521 (2010).

[29] D. Futturrer, J. Swiebodzinski, M. Governale, and J. König, Phys. Rev. B 87, 014509 (2013); D. Futturrer, M. Governale, and J. König, EPL 91, 47004 (2010).

[30] R. Fazio and R. Raimondi, Phys. Rev. Lett. 80, 2913 (1998); Phys. Rev. Lett. 82, 4950 (1999).

[31] P. Schwab and R. Raimondi, Phys. Rev. B 59, 1637 (1999).

[32] M. Krawiec and K.I. Wysokiński, Supercond. Sci. Technol. 17, 103 (2004).

[33] A.A. Clerk, V. Ambegaokar, and S. Hershfield, Phys. Rev. B 61, 3555 (2000).

[34] J.C. Cuevas, A. Levy Yeyati, and A. Martin-Rodero, Phys. Rev. B 63, 094515 (2001).

[35] Y. Yamada, Y. Tanaka, and N. Kawakami, Phys. Rev. B 84, 075484 (2011).

[36] Y. Avishai, A. Golub, and A.D. Zaikin, Phys. Rev. B 63, 134515 (2001); Y. Avishai, A. Golub, and A.D. Zaikin, Phys. Rev. B 67, 041301(R) (2003); T. Aono, A. Golub, and Y. Avishai,, Phys. Rev. B 68, 045312 (2003).

[37] A. Oguri, Y. Tanaka, and J. Bauer, Phys. Rev. B 87, 075432 (2013).

[38] T. Domański and A. Donabidowicz Phys. Rev. B 78, 073105 (2008); T. Domański, A. Donabidowicz, and K.I. Wysokiński, Phys. Rev. B 78, 144515 (2008); Phys. Rev.
B 76, 104514 (2007).

[39] C. Karrasch, A. Oguri, and V. Meden, Phys. Rev. B 77, 024517 (2008); C. Karrasch and V. Meden, Phys. Rev. B 79, 045110 (2009).

[40] A. Koga, Phys. Rev. B 87, 115409 (2013).

[41] K. Kang, Phys. Rev. B 58, 9641 (1998); S.Y. Cho, K. Kang, and C.-M. Ryu, Phys. Rev. B 60, 16874 (1999).

[42] Q.-F. Sun, J. Wang, and T.-H. Lin, Phys. Rev. B 59, 3831 (1999); Q.-F. Sun, H. Guo, and T.-H. Lin, Phys. Rev. Lett. 87, 176601 (2001).

[43] K. Levin, Q. Chen, C.-C. Chien, and Y. He, Annals of Physics 325, 233 (2010); T. Domański and J. Ranninger, Phys. Rev. Lett. 91, 255301 (2003).

[44] Y. Meir, N.S. Wingreen, and P.A. Lee, Phys. Rev. Lett. 66, 3048 (1991).

[45] J. Barański and T. Domański, Phys. Rev. B 84, 195424 (2011).

[46] L.I. Glazman and K.A. Matveev, Zh. Eksp. Teor. Fiz. Pis'ma Red. 49, 570 (1989) [JETP Lett. 49, 659 (1989)].

[47] S. Droste, S. Andergassen, and J. Splettstoesser, J. Phys.: Condens. Matter 24, 415301 (2012).

[48] A. Cottet, T. Kontos, and A. Levy Yeyati, Phys. Rev. Lett. 108, 166803 (2012). 